\title{
Mathematical Modeling of Exudative Retinal Detachment
}

\author{
Ram Avtar, Swati Srivastava* \\ Department of Mathematics, Harcourt Butler Technical University, Kanpur, India \\ Email: ^feb13swati@gmail.com
}

How to cite this paper: Avtar, R. and Srivastava, S. (2017) Mathematical Modeling of Exudative Retinal Detachment. Applied Mathematics, 8, 44-56.

http://dx.doi.org/10.4236/am.2017.81005

Received: December 7, 2016

Accepted: January 21, 2017

Published: January 24, 2017

Copyright $\odot 2017$ by authors and Scientific Research Publishing Inc. This work is licensed under the Creative Commons Attribution-NonCommercial International License (CC BY-NC 4.0). http://creativecommons.org/licenses/by-nc/4.0/

\begin{abstract}
A simple mathematical model for the exudative retinal detachment has been developed. The model takes into consideration a typical retinal blister with unknown shape and the fluid flow caused by its accumulation in the subretinal space through the fluid-leakage into the subretinal space from the choriocapillar is across the outer blood-retina barrier described by Darcy's law. The theory of bending beams is applied to model the deformation of the retinal blister. The boundary value problem describing the retinal deformation in dimensionless form is solved using the perturbation method. The computational results for the retinal deformation are presented through graphs to illustrate the sensitivity of the deformation to the elastic modulus, the moment of inertia and intraocular pressure and discussed.
\end{abstract}

\section{Keywords}

Retinal Pigment Epithelium, Subretinal Space, Choriocapillaris, Vitreous Humor

\section{Introduction}

The multi-layered retina is an effective transducer and functions as a light-receptor. The retina is connected to the vitreous body through its internal limiting membrane layer and with the choroid through the Bruch's membrane. The outer surface of the neurosensory retina is apposite to the retinal pigment epithelium, an outermost layer of the retina. In most of the area/region, the retinal pigment epithelium and the sensory retina are easily separated to form the subretinal space but the spread of subretinal fluid (SRF)/space is limited because the sensory retina and the pigment epithelium are firmly bound together at the optic disc and the ora serrata. The vitreous humor, liquefied vitreous and a fluid leaking outside the blood vessels of retina and/or the choroid percolating into the 
subretinal space may accumulate in the subretinal space. The accumulation of fluid in the subretinal space separates the neurosensory retina from the retinal pigment epithelium. The separation can occur when the adhesion forces between the two layers are overpowered/overcome by other different forces. The ageing, cataract surgery, myopia and trauma are some of the most common factors that contribute to the process.

Retinal detachment, which is a significant cause of blindness, must be dealt/ considered as a serious medical problem/emergency. After its occurrence of flashes of light and/or floaters in the visual field precedes, a dark shadow interfering with vision is perceived by the patients. The peeling away of the neurosensory retina from the underlying retinal pigment epithelium may result in the retinal detachment which is an affliction/disorder affecting many people. In the initial stages, the detachment may be localized, but, in the case of lacking its prompt and proper treatment, this may lead to progressive vision loss and subsequent blindness.

The three types of detachment distinguished are: (i) Retinal rhegmatogenous detachment (RRD), in which a hole or tear or break in the thin retinal layer forms and allows vitreous humor to pass from the vitreous body into the subretinal space between the sensory retina and the retinal pigment epithelium, (ii) Retinal tractional detachment (TRD) which is caused by pulling the neurosensory retina away from the RPE by fibrovascular tissue, caused by trauma, inflammation or neovascularization, (iii) Retinal exudative (or serous) detachment (ERD) in which a blister forms without a retinal hole. Inflammation, injury, or vascular abnormalities (e.g. choroid disruption) cause(s) it. This leads to fluid build-up/accumulation in sub-retinal space without the presence of a hole, tear or break.

The fluid accumulation in the subretinal space is a common characteristic in all types of retinal detachment. Different mechanisms may be involved in different types of the detachments. A shrinkage in vitreous body results in vitreous contraction. A tear, or hole or break in neurosensory retina allows the flow of vitreous humor, liquefied vitreous into the subretinal space under the pressuredifferential across the retina and fluid accumulation occurs therein. Sometimes neurosensory retina is pulled off the retinal pigment epithelium by external traction forces. The seepage of fluid which leaks through the blood retina barrier into the subretinal space develops a fluid build-up in the interstitial space. The fluid accumulated in the subretinal space pushes away the neurosensory retina which results in the retinal detachment. The retinal tension forces contribute to the detachment.

Under some pathological conditions, the fluid leakage from the retinal veins and/or choriocapillaris into the subretinal space exceed the fluid flow away/ outward actively pumped by the retinal pigment epithelium layer and a blister may result between the retina and the retinal pigment epithelium layer without a retinal tear. The interposed blister of accumulated subretinal fluid prevents the retina from its properly functioning. This situation causes an exudative retinal detachment, also called a serous retinal detachment. The failure of the retinal 
pigment epithelium actively pumping away of subretinal fluid, the increased/ enhanced substrate leakiness across the outer blood retina barrier due to the inflammation of the choriocapillaris, the excessive leakage of the serum through the walls of retinal veins due to the blockage in the vessels outward into the subretinal space contribute to the development of the exudative retinal detachment. Besides, some other factors such as the vitreous forces that pull the retina from the retinal pigment epithelium layer, may have some role in the detachment.

The retinal detachment is an ocular disorder which is well established and various therapeutic methods for its treatment have been evolved and are available. Despite numerous studies, its mechanism is not clearly elucidated and adequately described. In addition to experimental studies, several mathematical models [1] [2] for the retinal detachment propagation have been developed and analyzed. Mathematical modeling and simulation of the process may enrich/ enhance the present understanding of the retinal detachment.

Fitt et al. [1] proposed a post re-attachment retinal re-detachment problem by modeling the retina as an elastic layer and investigated its deformation under a prescribed wall shear stress. F. Baino mainly focused on the treatment of retinal detachment and examined the role and features of the materials used in vitreoretinal surgery, emphasizing scleral buckling and short-term/long-term vitreous tamponade [3]. Bottega et al. [4] developed a mechanics based mathematical model for retinal detachment treating the retina as an elastic shell while RPEchoroid-sclera as a rigid structure and concluded that the presence and size of a retinal tear or hole can have a stabilizing effect with regard to detachment propagation, under appropriate conditions. An improved analysis of the mechanical behavior of a detaching retina has been described by Bottega et al. and they revealed critical characteristic behavior of detachment propagation [5].

The present paper is concerned with the formulation of a mathematical model for the exudative retinal detachment. The model takes into consideration the fluid seepage into the subretinal space from the choriocapillaris and/or retinal veins and active outward pumping of the subretinal fluid by the retinal pigment epithelium. The retinal blister of accumulated subretinal fluid between retina and retinal pigment epithelium is approximated as a spherical section.

\section{Model Development}

A typical location of retinal blister is considered for the mathematical treatment of the exudative retinal detachment. In this type of the detachment, a retinal blister is formed between the retina and the RPE layer due to the subretinal fluid accumulation without the retinal tear. In view of the inward propagation of the retinal blister and its possible slow spread along the interface between the sensory retina and the retinal pigment epithelium, the flow of the subretinal fluid is assumed as a steady-state two dimensional flow. The subretinal fluid is treated as an incompressible Newtonian fluid with constant viscosity $\mu$ and density, $\rho$. The retinal pigment epithelium is treated as a fixed plane and is represented by the $\mathrm{x}$-axis $(z=0)$ and the retinal blister (considered as a thin flap) is assumed to 
be extended between $-L$ to $L$ and is described by $z=H(x)$. Due to the axisymmetry of the flow and detachment propagation, the domain of the problem is considered as: $z=H(x) ; 0 \leq x \leq L$ (Figure 1).

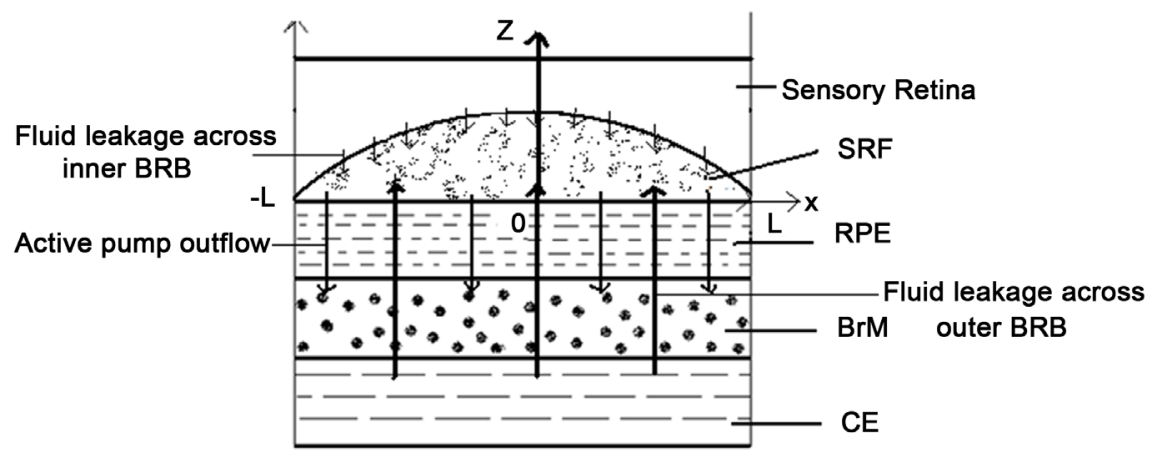

Figure 1. Schematic diagram of retinal blister. (SRF: Subretinal fluid; RPE: Retinal pigment epithelium; BrM: Bruch's membrane; CE: Choriocapillaris endothelium; BRB: Blood retina barrier; $\mathrm{H}(\mathrm{x})$ : Displacement of neurosensory retina from RPE layer $\mathrm{z}=0$ ).

\subsection{Governing Equations}

Applying the lubrication theory approximations to the Navier-Stokes equations and continuity equation for the slow flow of subretinal fluid between the retinal blister and the RPE layer, the governing equations of the problem reduce to the form:

$$
\begin{gathered}
\mu U_{z z}=P_{x} \\
P_{z}=0 \\
U_{x}+W_{z}=0
\end{gathered}
$$

where $(U, V, W)$ are the fluid velocity components, $\left(P_{x}, P_{y}, P_{z}\right)$ are the hydrodynamic pressure gradients in $x, y, z$ direction, respectively and $\mu$ corresponds to the dynamic viscosity.

\subsection{Boundary Conditions}

$$
\begin{gathered}
U(x, 0)=-\left.\frac{\sqrt{K}}{\sigma} \frac{\partial U}{\partial Z}\right|_{Z=0} \\
U(x, H(x))=0 \\
W(x, 0)=W_{0}(x) \\
W(x, H(x))=0 \\
P(x=0)=\Delta P \\
P(x=L)=0
\end{gathered}
$$

where $K$ is the permeability and $\sigma$, the slip parameter of the outer blood retina barrier.

\subsection{Solution to the Problem}

Solving Equation (1) subjecting to the boundary conditions (4i) and (4ii) we 
have,

$$
U=\frac{P_{x}}{2 \mu} 2\left[Z^{2}+\frac{H^{2}}{\left(\frac{\sqrt{K}}{\sigma}-H\right)}\left\{Z-\frac{\sqrt{K}}{\sigma}\right\}\right]
$$

From Equation (3) with boundary condition (5ii), we have,

$$
\begin{aligned}
W= & \frac{P_{x x}}{2 \mu}\left(\frac{H^{3}-Z^{3}}{3}\right)+\frac{P_{x x}}{2 \mu} \frac{H^{2}}{\left(\frac{\sqrt{K}}{\sigma}-H\right)}\left[\frac{H^{2}-Z^{2}}{2}+\frac{\sqrt{K}}{\sigma}(Z-H)\right] \\
& +\frac{P_{x} H H_{x}}{2 \mu}\left\{\frac{H^{2}-Z^{2}}{2}+\frac{\sqrt{K}}{\sigma}(Z-H)\right\}\left\{\frac{2\left(\frac{\sqrt{K}}{\sigma}-H\right)+H}{\left(\frac{\sqrt{K}}{\sigma}-H\right)^{2}}\right\}
\end{aligned}
$$

The seepage of the fluid across the blood-retina barrier into the subretinal space is described by the Darcy's law for the flow in porous media.

$$
W_{0}(x)=-\frac{K}{d \mu}\left(P_{C}-P\right)
$$

where $d$ is the width of blood-retina barrier and $P_{C}$, the fluid pressure in the choriocapillaris.

From boundary condition (5i),

$$
\begin{aligned}
W_{0}(x)= & \frac{P_{x x}}{2 \mu}\left(\frac{H^{3}}{3}\right)+\frac{P_{x x}}{2 \mu} \frac{H^{2}}{\left(\frac{\sqrt{K}}{\sigma}-H\right)}\left[\frac{H^{2}}{2}-\frac{\sqrt{K}}{\sigma} H\right] \\
& +\frac{P_{x} H H_{x}}{2 \mu}\left\{\frac{H^{2}}{2}-\frac{\sqrt{K}}{\sigma} H\right\}\left\{\frac{2\left(\frac{\sqrt{K}}{\sigma}-H\right)+H}{\left(\frac{\sqrt{K}}{\sigma}-H\right)^{2}}\right\}
\end{aligned}
$$

Comparing Equation (9) and (10),

$$
\begin{aligned}
-\frac{k}{d \mu}\left(P_{C}-P\right)= & \frac{P_{x x}}{2 \mu}\left(\frac{H^{3}}{3}\right)+\frac{P_{x x}}{2 \mu} \frac{H^{2}}{\left(\frac{\sqrt{K}}{\sigma}-H\right)}\left[\frac{H^{2}}{2}-\frac{\sqrt{K}}{\sigma} H\right] \\
& +\frac{P_{x} H H_{x}}{2 \mu}\left\{\frac{H^{2}}{2}-\frac{\sqrt{K}}{\sigma} H\right\}\left\{\frac{2\left(\frac{\sqrt{K}}{\sigma}-H\right)+H}{\left(\frac{\sqrt{K}}{\sigma}-H\right)^{2}}\right\}
\end{aligned}
$$

The position/displacement of the retinal blister depends on the pressure differential across it arising from the different pressures acting on the retinal blister. The theory of bending beams can be applied to describe/model the displacement/deformation of the retinal blister. The pressure differential across the retina is balanced by the intensity of the loading on it and the deformation of the retinal blister is described by a fourth order linear ordinary differential equation: 


$$
E I \frac{\mathrm{d}^{4} H}{\mathrm{~d} x^{4}}=P-P_{I}
$$

where $E$ and $I$ are the modulus of elasticity and the moment of inertia of retina, respectively.

In order to solve Equation (12) which is a fourth order linear equation, we must have appropriate boundary conditions that describe the constraint imposed by the hinges. We assume that the beam has a clamped end, therefore the boundary conditions are

$$
H_{x}(0)=0, H_{x x x}(0)=0, H(L)=H_{L}, H_{x}(L)=0
$$

First and last boundary conditions imply that both the ends of the retinal blister are horizontal. The assumption of no shearing force acts on the middle point of the blister is modeled by second boundary condition.

Rearranging Equation (12),

$$
\begin{gathered}
P=P_{I}+E I H_{x x x x} \\
P_{x}=E I H_{x x x x x} \\
P_{x x}=E I H_{x x x x x x}
\end{gathered}
$$

Using these values in Equation (11),

$$
\lambda H_{x x x x}=6 H_{x x x x x x}\left[\left(\frac{H^{3}}{3}\right)+\frac{H^{2}}{\left(\frac{\sqrt{K}}{\sigma}-H\right)}\left[\frac{H^{2}}{2}-\frac{\sqrt{K}}{\sigma} H\right]+\left\{\frac{H^{3}}{2}-\frac{\sqrt{K}}{\sigma} H^{2}\right\}\left\{\frac{2\left(\frac{\sqrt{K}}{\sigma}-H\right)+H}{\left(\frac{\sqrt{K}}{\sigma}-H\right)^{2}}\right\}\right]
$$

where $\lambda=12 \frac{k}{d}$.

The parameters can be non-dimensionalised using the scaling,

$$
x=L \bar{X}, P=P_{I}+\left(P_{L}-P_{I}\right) \bar{P}, H=L \bar{H}, k=L^{2} \bar{k}
$$

We now non-dimensionalise Equations (12) and (14) using the above nondimensional parameters,

$$
\frac{\mathrm{d}^{4} \bar{H}}{\mathrm{~d} \bar{x}^{4}}=-\alpha \bar{P}
$$

where $\alpha<0, \alpha=L^{3}\left(\frac{P_{I}-P_{L}}{E I}\right)$

And

$$
\lambda \bar{H}_{x x x x}=6 L \bar{H}_{x x x x x x}\left[\left(\frac{\bar{H}^{3}}{3}\right)+\frac{\bar{H}^{2}}{\left(\frac{\sqrt{\bar{K}}}{\sigma}-\bar{H}\right)}\left[\frac{\bar{H}^{2}}{2}-\frac{\sqrt{\bar{K}}}{\sigma} \bar{H}\right]+\left\{\frac{\bar{H}^{3}}{2}-\frac{\sqrt{\bar{K}}}{\sigma} \bar{H}^{2}\right\}\left\{\frac{2\left(\frac{\sqrt{\bar{K}}}{\sigma}-\bar{H}\right)+\bar{H}}{L\left(\frac{\sqrt{\bar{K}}}{\sigma}-\bar{H}\right)^{2}}\right\}\right]
$$

with boundary conditions,

$$
\begin{aligned}
& \bar{H}_{x}(0)=0, \bar{H}_{x x x}(0)=0, \bar{H}_{x x x x}(0)=\alpha\left(\frac{P_{I}-\Delta P}{P_{L}-P_{I}}\right), \\
& \bar{H}(1)=0, \bar{H}_{x}(1)=0, \bar{H}_{x x x x}(1)=\alpha\left(\frac{P_{I}}{P_{L}-P_{I}}\right)
\end{aligned}
$$


Equation (16) is a nonlinear 6th order differential equation and in order to solve it we need six boundary conditions. Equation (13) shows that we already have four boundary conditions, so we need two more. Thus we rearrange and non-dimensionalise the boundary conditions in (6i) and (6ii), to give the two more conditions.

Dropping bars for convenience from here onwards.

For $\lambda<1$,

Now solve the Equation (16) by using regular perturbation method where we assume that,

$$
H(x)=H_{0}(x)+\lambda H_{1}(x)+\cdots
$$

Using Equation (18) in Equations (16) and (17) we have,

$$
\begin{aligned}
& \lambda\left(H_{0}(x)+\lambda H_{1}(x)+\cdots\right)_{x x x x} \\
& =6 L\left(H_{0}(x)+\lambda H_{1}(x)+\cdots\right)_{x x x x x x}
\end{aligned}
$$

$$
\begin{aligned}
& {\left[\frac{\left(H_{0}(x)+\lambda H_{1}(x)+\cdots\right)^{3}}{3}+\frac{\left(H_{0}(x)+\lambda H_{1}(x)+\cdots\right)^{2}}{\left\{\frac{\sqrt{K}}{\sigma}-\left(H_{0}(x)+\lambda H_{1}(x)+\cdots\right)\right\}}\right.} \\
& {\left[\frac{\left(H_{0}(x)+\lambda H_{1}(x)+\cdots\right)^{2}}{2}-\frac{\sqrt{K}}{\sigma}\left(H_{0}(x)+\lambda H_{1}(x)+\cdots\right)\right]} \\
& +\left\{\frac{\left(H_{0}(x)+\lambda H_{1}(x)+\cdots\right)^{3}}{2}-\frac{\sqrt{K}}{\sigma}\left(H_{0}(x)+\lambda H_{1}(x)+\cdots\right)^{2}\right\} \\
& \left.\left\{\frac{2\left(\frac{\sqrt{K}}{\sigma}-\left(H_{0}(x)+\lambda H_{1}(x)+\cdots\right)\right)+\left(H_{0}(x)+\lambda H_{1}(x)+\cdots\right)}{L\left(\frac{\sqrt{K}}{\sigma}-\left(H_{0}(x)+\lambda H_{1}(x)+\cdots\right)\right)^{2}}\right\}\right]
\end{aligned}
$$

with boundary conditions,

$$
\begin{aligned}
& \left(H_{0}\right)_{x}(0)+\lambda\left(H_{1}\right)_{x}(0)+\cdots=0, \\
& \left(H_{0}\right)_{x x x}(0)+\lambda\left(H_{1}\right)_{x x x}(0)+\cdots=0, \\
& \left(H_{0}\right)_{x x x x}(0)+\lambda\left(H_{1}\right)_{x x x x}(0)+\cdots=\alpha\left(\frac{P_{I}-\Delta P}{P_{L}-P_{I}}\right), \\
& \left(H_{0}\right)(1)+\lambda\left(H_{1}\right)(1)+\cdots=0, \\
& \left(H_{0}\right)_{x}(1)+\lambda\left(H_{1}\right)_{x}(1)+\cdots=0, \\
& \left(H_{0}\right)_{x x x x}(1)+\lambda\left(H_{1}\right)_{x x x x}(1)+\cdots=\alpha\left(\frac{P_{I}}{P_{L}-P_{I}}\right)
\end{aligned}
$$


Comparing for zero order we have,

$$
\begin{aligned}
0=6 L\left(H_{0}\right)_{x x x x x x} & \frac{1}{3} H_{0}^{3}+\frac{H_{0}^{2}}{\left(\frac{\sqrt{K}}{\sigma}-H_{0}\right)}\left\{\frac{1}{2} H_{0}^{2}-\frac{\sqrt{K}}{\sigma} H_{0}\right\} \\
& \left.+\left\{\frac{1}{2} H_{0}^{3}-\frac{\sqrt{K}}{\sigma} H_{0}^{2}\right\}\left\{\frac{2\left(\frac{\sqrt{K}}{\sigma}-H_{0}\right)+H_{0}}{L\left(\frac{\sqrt{K}}{\sigma}-H_{0}\right)^{2}}\right\}\right]
\end{aligned}
$$

It means either

$$
6 L\left(H_{0}\right)_{x x x x x x}=0
$$

Or

$$
\frac{1}{3} H_{0}^{3}+\frac{H_{0}^{2}}{\left(\frac{\sqrt{K}}{\sigma}-H_{0}\right)}\left\{\frac{1}{2} H_{0}^{2}-\frac{\sqrt{K}}{\sigma} H_{0}\right\}+\left\{\frac{1}{2} H_{0}^{3}-\frac{\sqrt{K}}{\sigma} H_{0}^{2}\right\}\left\{\frac{2\left(\frac{\sqrt{K}}{\sigma}-H_{0}\right)+H_{0}}{L\left(\frac{\sqrt{K}}{\sigma}-H_{0}\right)^{2}}\right\}=0
$$

Solving Equation (20) with boundary conditions (19),

$$
\begin{aligned}
H_{0}(x)= & \frac{\alpha}{120}\left(\frac{\Delta P}{P_{L}-P_{I}}\right) x^{5}-\frac{\alpha}{24}\left(\frac{\Delta P-P_{I}}{P_{L}-P_{I}}\right) x^{4} \\
& +\frac{\alpha}{48}\left(\frac{3 \Delta P-4 P_{I}}{P_{L}-P_{I}}\right) x^{2}+\frac{\alpha}{240}\left(-7 \Delta P+10 P_{I}\right)
\end{aligned}
$$

Substituting (22) into Equation (18) we have,

$$
\begin{aligned}
H(x)= & \frac{\alpha}{120}\left(\frac{\Delta P}{P_{L}-P_{I}}\right) x^{5}-\frac{\alpha}{24}\left(\frac{\Delta P-P_{I}}{P_{L}-P_{I}}\right) x^{4}+\frac{\alpha}{48}\left(\frac{3 \Delta P-4 P_{I}}{P_{L}-P_{I}}\right) x^{2} \\
& +\frac{\alpha}{240}\left(-7 \Delta P+10 P_{I}\right)+O(\lambda)
\end{aligned}
$$

\section{Results and Discussions}

The sensitivity of the exudative retinal detachment to the elastic modulus and moment of inertia of the retina has been illustrated through the curves in Figures 2(a)-(c) and Figures 3(a)-(c). Also the effect of intraocular pressure on the detachment has been presented through the graphs in Figure 4 (Table 1).

It is observed from the graphs in Figures 2(a)-(c) that the deformation profiles of retinal blister is not linear and it decreases with an increase in the value of elastic modulus. It suggests that a decrease in the elasticity of the neurosensory retina tends to retard the exudative retinal detachment.

It is evident from the graphs in Figures 3(a)-(c) that the deformation/displacement of retinal blister is decreased with an increase in the moment of inertia. It illustrates that an increased value of moment of inertia of the sensory retina tends to decrease/retard the exudative retinal detachment. 

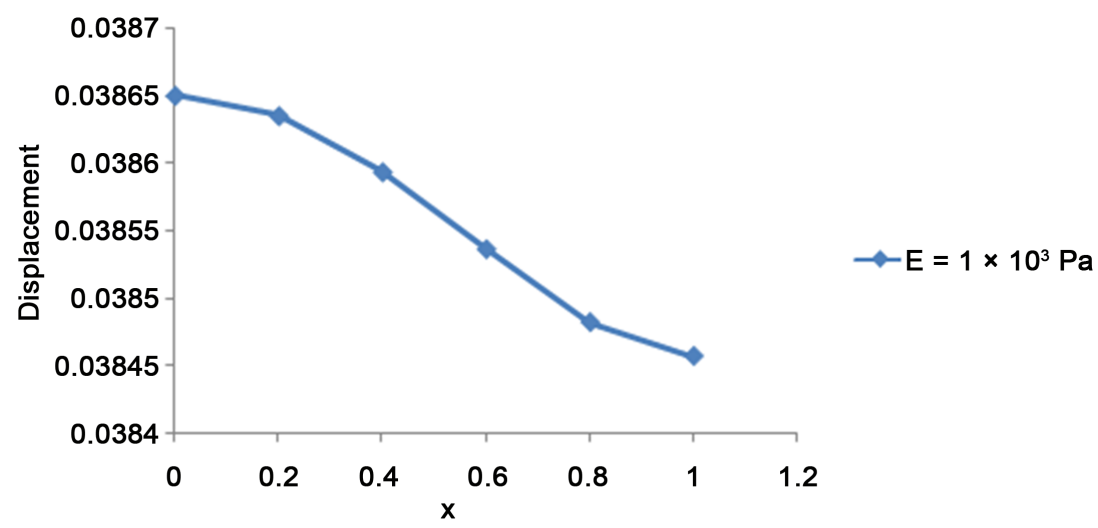

(a)

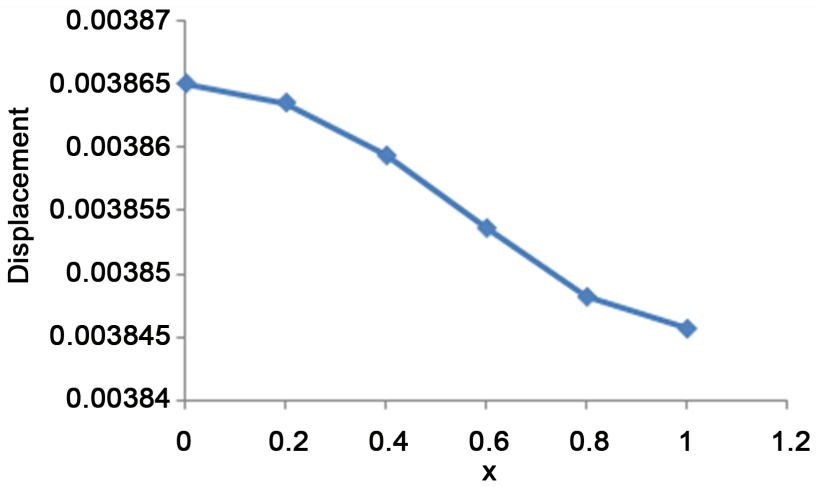

(b)

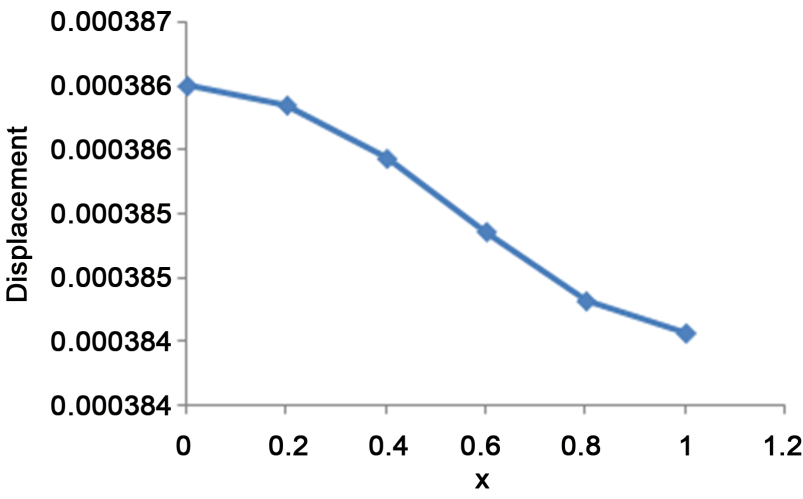

(c)

Figure 2. Effect of elastic modulus on displacement.

Table 1. Parameters appearing in the model and their approximate corresponding values in the retina.

\begin{tabular}{ccc}
\hline Parameter & Description & Typical physiological value \\
\hline $\mathrm{L}$ & Length of the detached retina & $1 \mathrm{~mm}^{*}$ \\
$P_{C}$ & Vascular pressure & $15-25 \mathrm{mmHg}^{\star *}$ \\
$P_{I}$ & Intraocular pressure & $10-20 \mathrm{mmHg}^{* *}$ \\
E & Retinal Yung's modulus & $10^{4} \mathrm{~Pa}{ }^{* *}$ \\
I & Moment of inertia & $4.14 \times 10^{-7} \mathrm{~kg} / \mathrm{m}^{2}$ \\
\hline
\end{tabular}

*Estimated and used by Z. Ismail [9]; ${ }^{* * E s t i m a t e d ~ a n d ~ u s e d ~ b y ~ T . ~ C h o u ~[2] ~}$ 


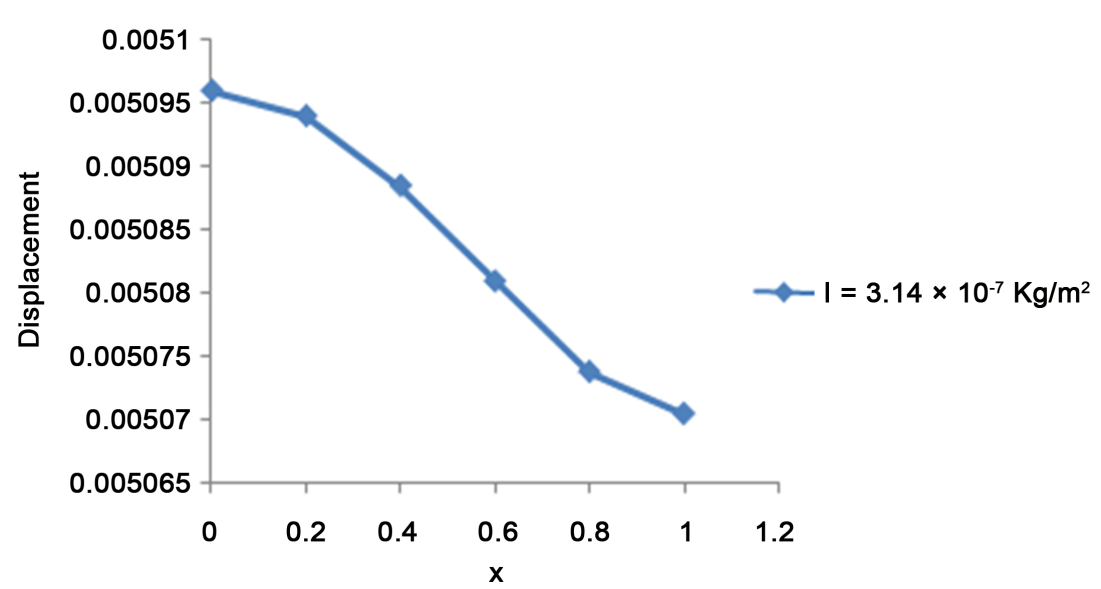

(a)

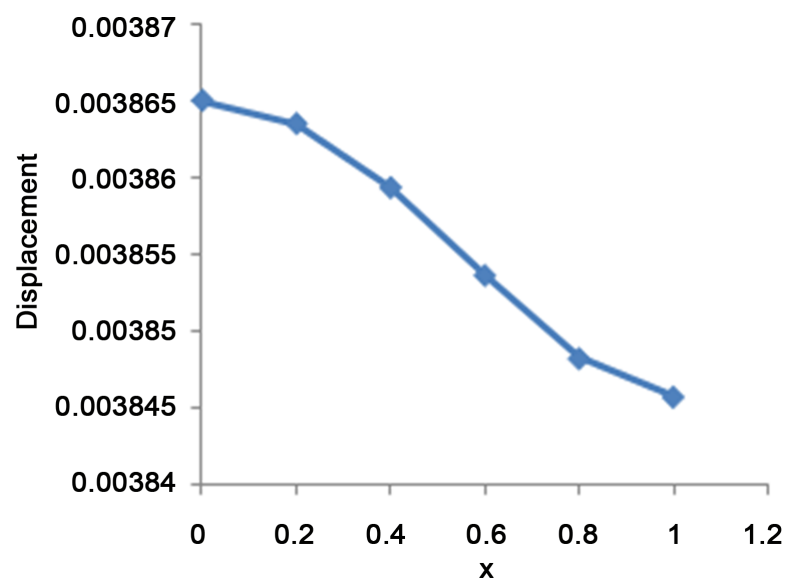

(b)

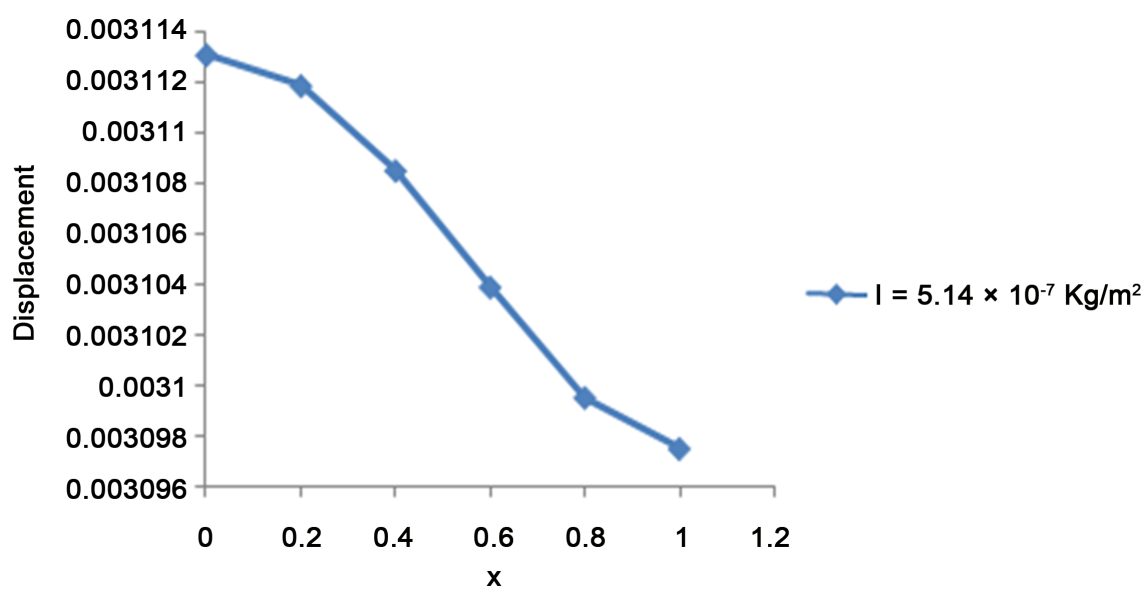

(c)

Figure 3. Effect of moment of inertia on displacement.

The effect of intraocular pressure on the deformation of retinal blister has been depicted/displayed in Figures 4(a)-(c). An observation of the curves demonstrates that an increase in the intraocular pressure causes an increase in the displacement. Thus, a rise in the intraocular pressure results in an increase in the retinal detachment. 

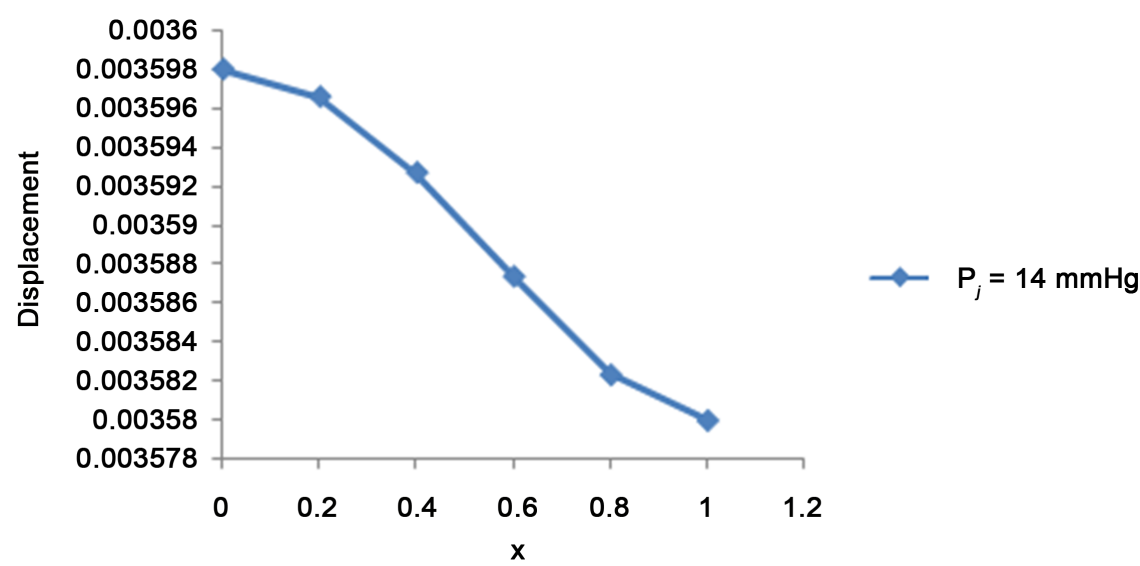

(a)

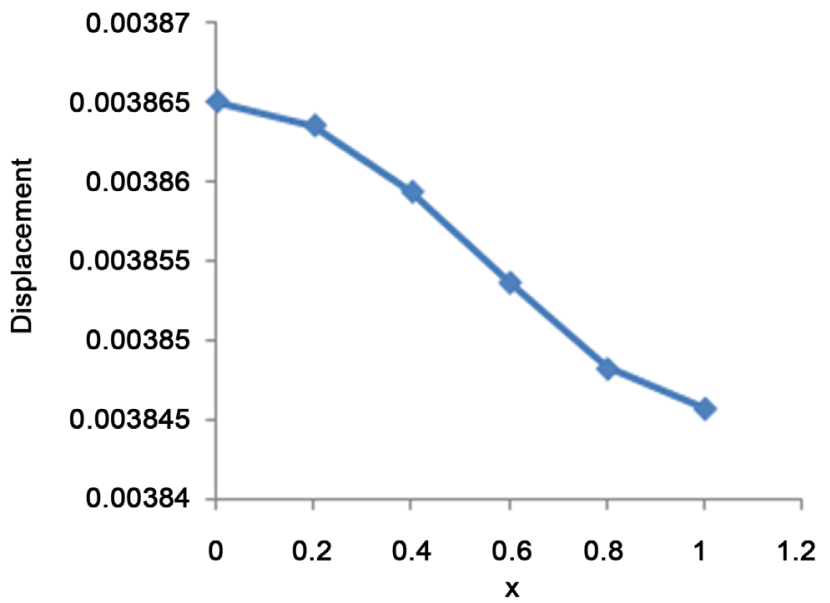

(b)

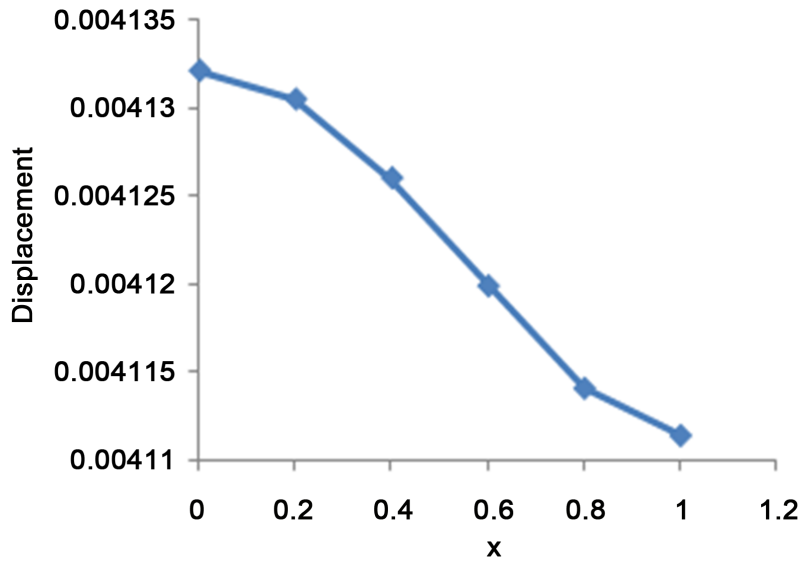

(c)

Figure 4. Effect of intraocular pressure on displacement.

\section{Conclusion}

A more realistic scenario involves a localized retinal detachment, or blister, under which subretinal fluid has accumulated. In view of the observations of the computational results of the deformation/ shape of the retinal blister, it is suggested that any therapeutic procedure that tends to decrease the elasticity of the 
retina and/or to increase the moment of inertia of retina and/or to elevate the intraocular pressure may help to treat the exudative retinal detachment. This result is supported by a number of experimental observations [6]. The work presented in this chapter is concerned only with the determination of shape of retinal blister and an analysis of its sensitivity to the elastic modulus and moment of inertia and the intraocular pressure. Results for modulus as a mechanism to close the detached area suggest the influence of the parameters of the retinal on its effectiveness with regard to closure and minimizing induced ocular pressure [7] [8]. We do not claim that great numerical accuracy should be attributed to the calculated value of modulus, since the real retina, though possessing elastic properties, is not a homogenous structure. Accurate measurements of height and applied force are difficult to obtain in a consistent way due to this inhomogeneity of the retinal material. This work can be extended to investigate theoretically the effect of permeability characteristics of outer and inner bloodretinas, of the choriocapillaris fluid pressure and active pump flow characteristic on the exudative retinal detachment in near future.

\section{Acknowledgements}

The authors are gratefully acknowledged to Dr. Rashmi Srivastava (Department of Mathematical Sciences and Computer Application, Bundelkhand University, Jhansi) for the constructive suggestions in view of which the work has been improved.

\section{References}

[1] Fitt, A. (2004) Post Re-Attachment Retinal Re-Detachment. Report on a Problem Studied at the UK Mathematics-in-Medicine Study Group, Strathclyde.

[2] Chou, T. and Siegel, M. The Mechanics of Retinal Detachment. Proceedings of the National Academy of Sciences of the United States of America.

[3] Baino, F. (2010) The Use of Polymers in the Treatment of Retinal Detachment: Current Trends and Future Perspectives. Polymers, 2, 286-322. https://doi.org/10.3390/polym2030286

[4] Bottega, W.J., Bishay, P.L., Prenner, J.L. and Fine, H.F. (2013) On the Mechanics of a Detaching Retina. Mathematical Medicine and Biology, 30, 287-310. https://doi.org/10.1093/imammb/dqs024

[5] Lakawicz, J.M., Bottega, W.J., Prenner, J.L. and Fine, H.F. (2015) An Analysis of the Mechanical Behaviour of a Detaching Retina. Mathematical Medicine and Biology, 32, 137-161. https://doi.org/10.1093/imammb/dqt023

[6] Jones, I.L., Warner, M. and Stevens, J.D. (1992) Mathematical Modelling of the Elastic Properties of Retina: A Determination of Young's Modulus. Eye, 6, 556-559. https://doi.org/10.1038/eye.1992.121

[7] Faude, F., Francke, M., Makarov, F., Schuck, J., Gartner, U., Reichelt, W., Wiedemann, P., Wolburg, H. and Reichenbach, A. (2001) Experimental Retinal Detachment Causes Widespread and Multilayered Degeneration in Rabbit Retina. Journal of Neurocytology, 30, 379-390. https://doi.org/10.1023/A:1015061525353

[8] Liu, X.Y., Wang, L.Z., Wang, C., Sun, G.Y., Liu, S.Y. and Fan, Y.B. (2013) Mechanism of Traumatic Retinal Detachment in Blunt Impact: A Finite Element Study. 
Journal of Biomechanics, 46, 1321-1327.

https://doi.org/10.1016/j.jbiomech.2013.02.006

[9] Ismail, Z. (2013) The Mathematical Modeling of Flow and Deformation in the Human Eye. Doctoral Thesis, University of Southampton, Southampton.

Submit or recommend next manuscript to SCIRP and we will provide best service for you:

Accepting pre-submission inquiries through Email, Facebook, LinkedIn, Twitter, etc. A wide selection of journals (inclusive of 9 subjects, more than 200 journals)

Providing 24-hour high-quality service

User-friendly online submission system

Fair and swift peer-review system

Efficient typesetting and proofreading procedure

Display of the result of downloads and visits, as well as the number of cited articles Maximum dissemination of your research work

Submit your manuscript at: http://papersubmission.scirp.org/

Or contact am@scirp.org 